\title{
MedienPädagogik
}

Zeitschrift für Theorie und Praxis der Medienbildung

\section{Fallstudie zur Informationskompetenz beim Lernen mit neuen Medien}

\author{
Udo Hinze und Gerold Blakowski
}

\section{1. $\quad$ Einleitung}

In der Debatte um die Medienkompetenz werden zunehmend die neuen Medien als unterstützende Mittel im E-Learningprozess thematisiert. Das selbstgesteuerte und flexible E-Learning ist auf eine hohe Handlungskompetenz der Lernenden angewiesen. Diese umfasst insbesondere die Fähigkeit, Informationen zu recherchieren, zu evaluieren und zu reduzieren. In einer Fallstudie wird exemplarisch aufgezeigt, wie sich divergente Kompetenz im Umgang mit Informationen manifestiert. Außerdem werden die Auswirkungen auf den Lernerfolg, die Lernzufriedenheit und die Nutzungsmodi der Medien dargestellt.

\section{Medienkompetenz}

Medienkompetenz ist trotz oder gerade wegen der ubiqitären Verwendung des Begriffes und der über 100 Definitionen, die Gapski (2001a) anführen konnte, ein Schlagwort, dessen Präzisierung nicht immer erfolgt oder überzeugt. Ist Medienkompetenz damit nur ein Containerwort und ein „ebenso pompöses wie rätselhaftes Ideal“ der Medienpädagogik (Kübler 1996)?

Die Kritik am Begriff Medienkompetenz zielt auf die Unbestimmtheit bei einer gleichzeitigen Beliebigkeit der Begriffsverwendung. Hier schließt die Debatte an den Diskurs um Kompetenz an. Kompetenz als ein „ungefährer“ Terminus (Orthey 2002) wurde im pädagogischen Kontext mit „bisweilen bizarr anmutenden Sinn-Konstruktionen“ (Wollersheim 1990, S. 92) verwendet. Weitgehender Konsens besteht darüber, das Kompetenz „Qualität von Wissen, die nicht inhaltlich oder sachlich bestimmt, sondern höher aggregiert ist“ (Dewe/Sander 1996, S. 128) darstellt. Die Deduktion konkreter Lernziele und -wege bleibt dabei vage. Gerade die Unschärfe eines holistischen Medienkompetenzbegriffes wird allerdings - analog zum Bildungsbegriff - als Chance interpretiert und gesehen. So sind nach Baacke (1999) die fehlenden inhaltlichen Vorgaben und didaktischen und methodischen Hilfen zur Umsetzung der Medienkompetenz immer auch als Entwicklungschance zu deuten. 
Komplementär zielt ein Teil der Kritik auf die konkrete Gebundenheit technokratischer Definitionen von Medienkompetenz an die jeweils aktuelle Medientechnologie und die Deduktion aus deren Konditionen (z.B. Kübler 1996). Medienkompetenz ist vielmehr eine „allgemeine Fähigkeit, die prinzipiell auf alle Medien bezogen ist“ (Aufenanger 2002, S.120). Festzuhalten bleibt aber, dass Medienkompetenz (wiederum analog zur Bildung) nicht zeitlos definiert, sondern nur im jeweiligen Kontext erschlossen werden kann. Das verdeutlichen schon die Unterschiede zwischen der Begriffsverwendung in Politik, Wirtschaft, Rechtssystem und in der Pädagogik (vgl. Gapski 2001b). Auch für die Nutzung konkreter Medien sind jeweils spezifische Fähigkeiten notwendig. Unter dezidierter Berücksichtigung der Spezifika neuer Medien differenziert daher Winterhoff-Spurk (1997) in Medienkompetenz und Informationskompetenz. Beide Kompetenzen sind Teilbereiche der übergeordneten Kommunikationskompetenz. Unter dem Begriff Medienkompetenz wird die Kompetenz zum Umgang mit den alten Massenmedien (Printmedien, Hörfunk und Fernsehen) verstanden. Für den Umgang mit den neuen Medien bzw. Informationstechnologien (PC und Internet) wird der Begriff Informationskompetenz vorgeschlagen.

\section{Informationskompetenz}

Mit neuen Medien lassen sich neue Informationen sehr leicht erstellen, distribuieren und referenzieren. Allerdings kann die Informationserzeugung und -distribution in quantitativ neuen Ausmaßen in Konsequenz zum oft zitierten „Information Overload“ (vgl. z.B. Gorman 2003) führen. Um dies zu vermeiden, wird der adäquate Umgang mit Informationen zu einer Kulturtechnik der Informationsgesellschaft.

Deutlich wird dies im Bereich E-Learning, wo der Zugang zu einer Vielzahl an Informationen potenziell ein zentraler Vorteil ist. Allerdings wird die ambivalente Wirkung der Informationsflut oft drastisch deutlich. Informationen in großen Umfang nutzen zu können, ist nicht per se positiv für den Lernerfolg. Mögliche negative Folgen sind insbesondere beim selbstgesteuerten E-Learning Phänomene wie „Lost in Hyperspace“ oder der „Serendipity-Effekt“ (Kuhlen 1991, S. 129). Dabei verliert der Lernende unter der kognitiven Überlast den roten Faden oder im Extremfall sogar das eigentliche Lernziel aus den Augen. Er fühlt sich überfordert und lernt - wenn überhaupt - eher zufällig.

Vor allem die Fähigkeit zur Informationsreduktion ist daher eine wesentliche Voraussetzung für ein weitgehend selbstgesteuertes und -organisiertes E-Learning. Die Reduktion kann durch Selektion und Informationsordnung, d.h. die Bündelung und Strukturierung von Information realisiert werden. Ein anderer Weg sind subjektive Entlastungsstrategien wie die Einschränkung der kognitiven Informationsverarbeitung oder die Problemvereinfachung (vgl. Hagge 1994). Diese komplexen Fähigkeiten im Umgang mit 
Informationen werden - in Anlehnung an die im angloamerikanischen Raum oft thematisierte „information literacy“ - als wesentliche Aspekte der Informationskompetenz konzeptualisiert. Das im Deutschen bislang eingeschränkte Begriffsverständnis von Informationskompetenz etwa als Fähigkeit zur adäquaten Benutzung von Bibliotheken wird dabei erweitert. Grundlegendes Problem ist beim E-Learning weniger die Suche nach Information sondern die Einordnung, Selektion und Reduktion. Darauf verweist die von Larsen (2001) angeführte, sehr spezifische Definition von Informationskompetenz:

- "know when they need information

- find information

- evaluate information

- process information

- use information to make appropriate decisions in their lives".

Neben dem Recherchieren sind also Fähigkeiten wie verifizieren, evaluieren, reduzieren, strukturieren, produzieren und präsentieren von Informationen gefordert (Borrmann/Gerdzen 1996).

\section{Fallstudie}

\subsection{Rahmenbedingungen}

Anhand eines Projektes zum kooperativen E-Learning wurde exemplarisch gezeigt, wie sich unterschiedliche Informationskompetenz beim E-Learning manifestiert. Dazu wurde anschließend an ein einsemestriges Seminar an der FH Stralsund zum Thema Computer Supported Cooperative Work bzw. Learning (CSCW/L) ein synchrones siebenstündiges kooperatives Lernszenario durchgeführt. Insgesamt agierten drei Gruppen mit jeweils drei Studierenden. Die Aufgabe beinhaltete die Erstellung einer Präsentation über den exemplarischen Einsatz von CSCW/L in einem global agierenden Unternehmen.

Ausgangspunkt war die Hypothese, dass sich die Divergenzen in der Informationskompetenz bei einer komplexen Aufgabe ohne einen dezidiert vorgegebenen Lösungsweg explizit manifestieren. Daher wurde der Aspekt der Selbststeuerung stark betont und die inhaltliche und formale Hilfe weitgehend eingeschränkt. Zur Kommunikation und Kooperation in den Lerngruppen und zur Dokumentation der einzelnen Arbeitsschritte wurden verschiedene Tools zur Verfügung gestellt.

1) Für die Aufgabenstrukturierung und zur Unterstützung kreativer Prozesse wurde das Kreativitätstool Mindmanager 3.5 bereitgestellt. Mindmanager baut auf der Mindmap-Technik auf (z.B. Buzan/Buzan 1995). Es besteht die Möglichkeit, in einer Konferenz kooperativ Mindmaps als verteilte Anwendungen zu erstellen. 
2) Als primäres Kommunikationsmittel wurde Videokonferenz genutzt. Gerade bei kurzfristiger Gruppenarbeit, die auf intensive Kooperation angewiesen ist, kann die notwendige und in der Regel umfangreiche synchrone Kommunikation sinnvoll und effizient über Videokonferenz realisiert werden.

3) Komplementär zur Videokonferenz war mit Netmeeting 3.01 textbasierte synchrone Kommunikation und Kooperation mit verteilten Anwendungen möglich.

4) Als kooperative Lernumgebung stand TeamWave 4.3 zur Verfügung. TeamWave war ein integriertes, auf der Raummetapher aufbauendes System, dass eine Reihe unterschiedlicher Kommunikations-, Kooperations- und Koordinationsmöglichkeiten beinhaltete. Hintergrund der Räume war jeweils ein Whiteboard, auf dem die Studierenden verteilt arbeiten konnten. Es waren drei Gruppenräume und eine „Bibliothek“ eingerichtet. In dieser bestand die Möglichkeit, weiterführende Informationen nachzuschlagen. Außerdem konnten die Lernenden individuell weitere Räume einrichten.

\subsection{Ablauf}

Der weitere Ablauf der Kooperation wurde von den Studierenden weitgehend autonom gestaltet. Da die Studierenden mit den Funktionalitäten und Einsatzgebieten der Tools vertraut waren, war z.B. die Möglichkeit, zunächst Aufgaben und Probleme mit Mindmanager zu strukturieren, als ein möglicher Einstieg in die Gruppenarbeit bekannt. Alle drei Gruppen begannen dementsprechend mit der Einrichtung von Mindmanagerkonferenzen und Videoverbindungen. In dieser Phase waren nur marginale Differenzen zwischen den Gruppen festzustellen. Bei funktionierender Videoverbindung war dann eine divergente Arbeitsweise der Gruppen festzustellen.

\subsubsection{Gruppe 1}

In der Gruppe 1 wurde über die gesamte Bearbeitungszeit die Mindmanagerkonferenz offen gehalten und fast bis zum Schluss inhaltlich an der Mindmap gearbeitet (vgl. Abb. 1). Dabei wurden die einzelnen Aspekte der Mindmap fast ausschließlich aufbauend auf dem im Seminar erworbenen Wissen erstellt. Die weiterführenden Informationen in der Bibliothek wurden nur als Ergänzung und erst in der Endphase der Gruppenarbeit genutzt. Eigene Recherche im Internet wurde nicht betrieben. Die Kooperation bei der Mindmaperstellung wurde durch eine intensive Kommunikation über Videokonferenz unterstützt.

Insbesondere über die einzelnen Vor- und Nachteile von CSCW wurde intensiv diskutiert. In der Regel wurde ein Konsens erzielt. Nur selten wurden Entscheidungen per Mehrheitsbeschluss gefällt. 


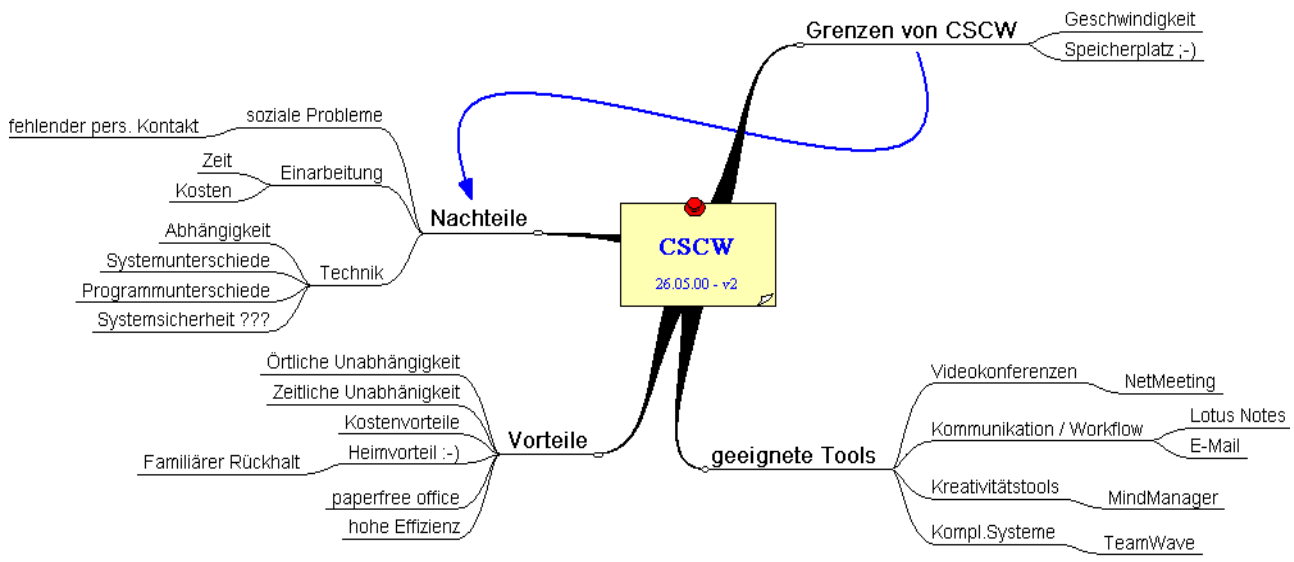

Abb. 1: Mindmap (Gruppe 1).

Das kooperative System TeamWave wurde vor allem zur Koordination genutzt. Ohne dass explizit ein Gruppenleiter bestimmt wurde, konzentrierten sich diese Arbeiten nicht im Gruppenraum, sondern wurden in einem Einzelraum durchgeführt. Es wurden vor allem, aufbauend auf den durch die Mindmap vorgegebenen Schwerpunkten, mögliche Ergänzungen zum Vortrag diskutiert. Dabei ging es um inhaltliche Aspekte (etwa die Bewertung von Informationen aus der Bibliothek) und die Gestaltung des Vortrages z.B. durch Bilder. Der eigentliche Gruppenraum wurde nur für die Vorbereitung der Videokonferenz und das Sammeln von Hauptpunkten für den Vortrag benötigt (vgl. Abb. 2).

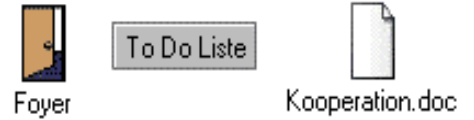

Aufgabe: Kooperation übergreifend organisieren
Lösungsschritte: CSCW

- konkrete Vor- und Nachteile durch CSCW

- Grenzen des CSCW

- Ein- und Umsetzung mit CscW

- Tools? i adäquate Umsetzung der

\author{
IP Stefan 194.94.75.206 \\ IP Annette 194.94.75.208 \\ IP Jens 194.94.75.181
}

Abb. 2: Strukturierter Gruppenarbeitsraum (Gruppe 1). 
Die Endfassung des Vortrages wurde nach vorheriger Absprache nur von einem Studierenden erstellt. Von den anderen Gruppenmitglieder wurde der Prozess via Netmeeting und Videokonferenz beobachtet und kommentiert.

Im abschließenden Vortrag, der aus einer kommentierten Slideshow bestand, wurden die vier in der Mindmap thematisierten Schwerpunkte aufgegriffen und näher erläutert. Dadurch war der Vortrag, trotzdem er nur aus 5 Folien bestand, sehr stringent. Allerdings wurde die konkrete Fallstudie inhaltlich nur wenig tangiert. Der zeitliche Rahmen der Kooperation wurde von allen Teilnehmern als angemessen beurteilt. Das lag vor allem daran, dass die Informationsmenge überschaubar gehalten wurde. Externe Informationen etwa aus dem Internet wurden nur im Rahmen der in der Bibliothek referenzierten Quellen analysiert.

\subsubsection{Gruppe 2}

Die Gruppe 2 brach die Mindmanagerkonferenz schon nach kurzer Zeit ergebnislos ab. Die Studierenden beschäftigten sich vor allem mit den Funktionalitäten von TeamWave und führten eine nur teilweise aufgabenbezogene Kommunikation mit Videokonferenz.

Obwohl in der Bibliothek weiterführende Informationen zu CSCW/L angegeben waren, konzentrierte sich die darauffolgende Arbeit der Studierenden auf die Recherche nach relevanten Beiträgen zum Thema im Internet. Diese wurden im Gruppenraum zusammengetragen. Dadurch ergab sich eine Fülle von Informationen, die relativ unreflektiert nebeneinander standen. Problematisch war darüberhinaus, das es sich teilweise um Hypertextdokumente wie etwa Linklisten handelte. Eine planmäßige Informationsevaluation war bei diesen Quellen in der vorgegebenen Zeit kaum möglich.

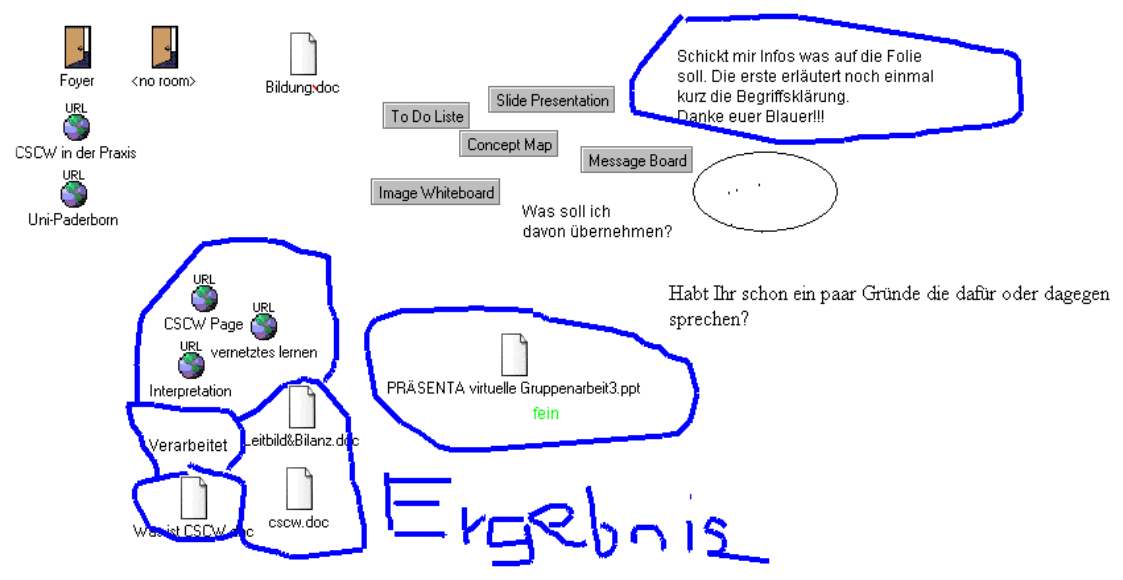

Abb. 3: Gruppenarbeitsraum (Gruppe 2). 
Die Diskussion, welche Informationen relevant für den Vortrag sind, wurde relativ intensiv geführt. Obwohl ebenfalls kein Gruppenleiter bestimmt war, wurde die definitive Entscheidung über die Präsentation - nur bedingt demokratisch - durch ein Gruppenmitglied zumindest forciert (vgl. Abb. 3).

Die Bearbeitung der Aufgabe war durch die Vielzahl unterschiedlicher Informationsquellen, die teilweise aus Hypertextdokumenten bestanden, erheblich erschwert. Eine sinnvolle Integration der Informationen in die Präsentation konnte nicht geleistet werden. Um trotzdem zu einem Ergebnis zu kommen, wurde der Entscheidungsprozess verkürzt. Die weitere Bearbeitung der Informationen wurde abgebrochen und es begann die Vorbereitung des Vortrages. Die Präsentation wurde inhaltlich weitgehend nur durch ein Gruppenmitgliedes erstellt. Im Gegensatz zur Gruppe 1 fand Kommunikation und Kooperation in dieser Phase nicht statt. Der Konsens bestand darin, dass die anderen Gruppenmitglieder (die sich bis zum Präsentationstermin vor allem mit der Gestaltung der Präsentation beschäftigten) die vorgeschlagene Lösung akzeptierten. Der Vortrag umfasste insgesamt sehr viele Folien, war aber weniger stringent und noch allgemeiner als der Vortrag von Gruppe 1. Der zeitliche Rahmen der Aufgabe wurde von zwei Gruppenmitgliedern als zu kurz, von einem als angemessen beurteilt.

\subsubsection{Gruppe 3}

Die Gruppe 3 beschäftigte sich ebenfalls fast nur mit Videokonferenz und TeamWave. Die Mindmanagerkonferenz wurde nach kurzer Zeit ergebnislos abgebrochen. Anfangs wurde vor allem die Videokonferenz genutzt. Danach wurde im Internet individuell nach Quellen zum Themengebiet CSCW/L recherchiert. Im Gegensatz zu den anderen Gruppen wurden die Ergebnisse in unterschiedlichen Räumen abgelegt. Der Gruppenraum, der prinzipiell für die Koordinierung der Einzelaktivitäten genutzt werden sollte, blieb leer. Durch die Vielzahl teilweise redundanter Informationen waren die Räume der Gruppenmitglieder mit sehr vielen Verweisen und Quellen belegt (vgl. Abb. 4).

Deutlich wird, dass auch Ergebnisse von Suchmaschinen (hier Altavista und Yahoo) einbezogen wurden. Diese waren in der vorgegebenen Zeit nicht annähernd individuell oder kooperativ zu bewerten. Die weitgehend planlose Informationssuche nahm insgesamt den Hauptteil der Zeit in Anspruch. Da die einzelnen Gruppenmitglieder jeweils primär die eigenen Informationsquellen sichteten und (soweit als möglich) bewerteten, war es schwierig, zu einem Gesamtergebnis zu gelangen. Dementsprechend wurde die Erstellung der Präsentation sehr spät und nur von einem Teilnehmer in Angriff genommen. Die anderen Teilnehmer akzeptierten das in Inhalt und Form nur suboptimale Ergebnis. Inhaltlich wurde sich auf allgemeine Definitionen und Klassifikationen beschränkt. Der Bezug zum eigentlichen Thema (Einsatz von CSCW/L im Vertrieb) war nicht vorhanden. Der Zeitrahmen wurde von allen Gruppenmitgliedern als zu gering eingeschätzt. 


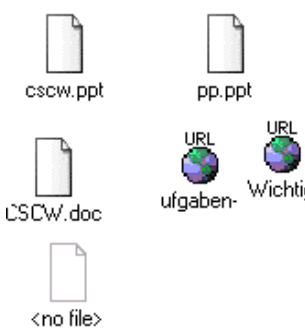

Entwicklung von CSCW-Systemen

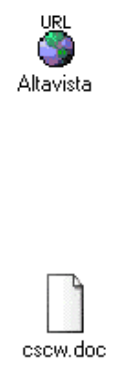

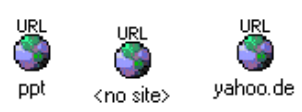

Sofort Offinen dann sehen wir glaube ich durchtllt!ll!

〈no site〉 yahoo.de

Abb. 4: „Information Overload“ im Einzelraum (Gruppe 3).

\subsection{Zusammenfassung}

In der Fallstudie wird schon visuell in den unterschiedlichen Nutzungsmodi der Tools (vgl. die Abb. 1-4) deutlich, wie sich Informationskompetenz im E-Learning manifestiert. In Gruppe 1 war die Informationskompetenz und damit auch die Fähigkeit, selbstgesteuert zu lernen, in relativ hohem Maße vorhanden. Auf die Einbeziehung externer Quellen wurde weitgehend verzichtet. Die bereitgestellten Informationen wurden umfassend diskutiert. Der Schwerpunkt lag weniger bei der Informationssuche, als vielmehr bei der Evaluation und Integration der Wissensquellen.

Die Gruppe 2 trug viele, auch externe Informationen gemeinsam zusammen. Die letztendliche Bewertung und Integration wurde aber nicht kooperativ und konsensual, sondern dominiert von einem Gruppenmitglied durchgeführt. Bei der kooperativen Bewertung und Selektion der Informationen zeigten sich deutliche Defizite. Dies lag an der Vielzahl von teilweise hyperstrukturierten Dokumenten.

In Gruppe 3 war die Aufgabe im Rahmen der additiven Herangehensweise in der vorgesehenen Zeit kaum zu lösen. Die Informationen wurden individuell recherchiert und evaluiert. Da der Anteil an Hypertextdokumenten sehr hoch war, wäre die Aufgabe auch bei einer intensiveren Kommunikation und Kooperation kaum zu lösen gewesen. Der Schwerpunkt lag für die Studierenden vor allem in der Informationssuche. Die Bewertung und Strukturierung der Informationen wurde nur ansatzweise versucht. Dieser Aspekt wurde für die Studierenden erst zum Ende der Gruppenarbeit virulent. Allerdings war zu diesem Zeitpunkt kaum noch eine sinnvolle Lösung möglich. Dementsprechend reduzierten die Studierenden das Problem auf den subjektiv zu kleinen Zeitkorridor. 


\section{Fazit}

Für die Fallstudie sind bei gleicher Aufgabenstellung und identischem technischen Setting erhebliche Unterschiede bei den Bearbeitungsmodi und Lernerfolgen zu konstatieren. Ursächlich dafür sind neben den didaktisch-methodischen und technischen Rahmenbedingungen auch die Kompetenzen zur Problemstrukturierung und Informationsreduzierung. Diese spielen unter den hohen Anforderungen, die selbstgesteuertes ELearning an die Lernenden stellt, eine zentrale Rolle. Damit ist Informationskompetenz keine Leerformel, sondern wie - hier auch visuell feststellbar - eine essentielle Voraussetzung für ein erfolgreiches selbstgesteuertes E-Learning.

Kurzfristig kann durch ein anderes methodisches Vorgehen reagiert werden. So kann der „Information Overload“ durch Strukturierung der Aufgabe oder eine intensivere Unterstützung und Intervention verhindert werden. Allerdings führt dies zu Nivellierung. Von einer stärkeren Strukturierung der Aufgabe profitieren die Lernenden mit niedrigen Lernvoraussetzungen mehr als diejenigen mit höherer Lernvoraussetzung (Webb/ Palincsar 1996). Außerdem vermindert die Strukturierung den Grad an Selbstorganisation und -steuerung im E-Learning. Damit werden die positiven Aspekte, die selbstgesteuertes Lernen prinzipiell hat (vgl. Dohmen 1999), relativiert.

Mittel- und langfristig sind daher Konzepte notwendig, die speziell die Fähigkeit zur gezielten Nutzung von Informationen fördern. In der Fallstudie war insbesondere die Fähigkeit unterschiedlich ausgeprägt, Informationen einzuordnen, selektieren und reduzieren zu können. Bei der Möglichkeit zur Förderung dieser Kompetenz, die in einem weiteren Kontext die zentrale Frage, „Wie wird man eigentlich medienkompetent?“ (Sander 2001) tangiert, kann auf die eingangs thematisierte Chance eines unbestimmten Medienkompetenzbegriffes verwiesen werden. Nach Baacke (1999) werden die fehlenden inhaltlichen Vorgaben in Projektarbeit prozessual erarbeitet. Beachtet man, dass auch der Erwerb von Informationskompetenz kein wirklich systematischer, sondern ein situationsbezogener Prozess ist (Cheuck 1999), dann ist eine sinnvolle Förderung nur und ausschließlich in Projekten realisierbar.

\section{Literatur}

Aufenanger, S.: Medienerziehung und Medienkompetenz. In: Gruber, T. (Hrsg.): Was bieten die Medien? Was braucht die Gesellschaft. Chancen und Risiken moderner Kommunikation. (Sonderpublikationen des Bayerischen Rundfunks Bd. 2). München 2002, S. 119-123.

Baacke, D.: Medienkompetenz als zentrales Operationsfeld von Projekten. In: Baacke, D. u.a. (Hrsg): Handbuch Medien: Medienkompetenz - Modelle und Projekte, Bonn 1999 (Bundeszentrale für politische Bildung), S.31- 35. hier zit nach [http://www.medienpaedagogik-online.de/ $\mathrm{mk} / 00381 /]$ (22.01.2003).

Borrmann, A./ Gerdzen, R.: Medienkompetenz, Informationskompetenz, Nutzungskompetenz. Kulturtechniken der Informationsgesellschaft. 1999. [http://www.linse.uni-essen.de/tagungen/ sdd/ gerdzen.htm] (10.08.2000).

Buzan, T./ Buzan, B.: The Mind Map Book. BBC Consumer Publishing 1995. 
Cheuck, B. W.: Rethinking information literacy education: appreciating human information seeking and use as a dynamic and situational process. IATUL News 8/1999, pp. 3-8.

Dewe, B./ Sander, U.: Medienkompetenz und Erwachsenenbildung. In: von Rein, A. (Hrsg.): Medienkompetenz als Schlüsselbegriff. Bad Heilbrunn 1996, S. 115-142.

Dohmen, G.: Weiterbildungsinstitutionen, Medien, Lernumwelten: Rahmenbedingungen und Entwicklungshilfen für das selbstgesteuerte Lernen. Bonn: Bundesministerium für Bildung und Forschung. 1999.

Gapski, H.: Medienkompetenz. Eine Bestandsaufnahme und Vorüberlegungen zu einem systemtheoretischen Rahmenkonzept. Wiesbaden 2001a.

Gapski, H.: Was ist Medienkompetenz? Vortrag auf der Fachtagung "Medienkritik und Medienkompetenz” am 25 September 2001 in Dortmund. [http://www.ecmc.de/fachtagung-medienkompetenz-2001/] (22.01.2003).

Gorman, M.: The enduring library: technology, tradition, and the quest for balance. Chicago 2003.

Hagge, K.: Informations-Design. Heidelberg 1994.

Kübler, H.-D.: Kompetenz der Kompetenz der Kompetenz ... Anmerkungen zur Lieblingsmetapher der Medienpädagogik. In: medien praktisch 2/1996, S.11-15.

Kuhlen, R.: Hypertext: ein nicht-lineares Medium zwischen Buch und Wissensbank. Berlin u.a. 1991

Larsen, L. L.: Information Literacy: The Web is not an Encyclopedia. 1991. [http://www.inform.umd. edu/LibInfo/literacy/] (19.11.2001).

Orthey, F. M. : Der Trend zur Kompetenz. Begriffsentwicklung und Perspektiven. In: Supervision, 1/2002, S. 7-14.

Sander, U.: Wie wird man eigentlich medienkompetent? In: Gesellschaft für Medienpädagogik und Kommunikationskultur (GMK) (Hrsg.): Medienkompetenz in Theorie und Praxis. Bielefeld 2001, S. 92-93.

Webb, N. M./ Palincsar, A. S.: Group processes in the classroom. In: Berlinger, D. C./ Calfee, R. C. (Eds.): Handbook of educational psychology. New York 1996, pp. 841-873.

Winterhoff-Spurk, P.: Medienkompetenz: Schlüsselqualifikation der Informationsgesellschaft? Medienpsychologie, 9 (3), 1997, S. 182-190.

Wollersheim, H.-W.: Kompetenz: Zur Verwendung des Begriffs in Pädagogik und Psychologie. In: Geißler, E. E. (Hrsg.): Bildung für das Alter - Bildung im Alter: Expertisensammlung. Bonn 1990, S. 75-100. 